\title{
LONG-TERM PROTECTION
}

Oral contraceptives reduce the risk of ovarian cancer, but how long does the protection last after use ceases? The Collaborative Group on Epidemiological Studies of Ovarian Cancer analysed data for 23,257 women with ovarian cancer and 87,303 without ovarian cancer from 45 epidemiological studies in 21 countries. The individuals were stratified by study, age, parity and hysterectomy, because these variables are related both to the use of oral contraceptives and to the risk of developing ovarian cancer.

Overall $31 \%$ cases and $37 \%$ controls had ever used oral contraceptives, for an average of $4-5$ years. The cancers were diagnosed in 1993, on average, and the mean age at diagnosis was 56 years. The longer a woman had used oral contraceptives, the greater the decrease in ovarian cancer risk, and this risk persisted for more than 30 years after use was stopped. The risk reduction was $29 \%$ for those who had stopped $<10$ years previously, 19\% for those stopping 10-19 years previously, and $15 \%$ for those stopping 20-29 years previously.

Oral contraceptives were first licensed for use almost 50 years ago. In the 1960 s concentrations of oestrogen in oral contraceptives were typically $100 \mu \mathrm{g}$ or more. By 1980 most preparations were low-dose - typically containing $30 \mu \mathrm{g}$ or less of oestrogen. Despite these changes, risk reductions for use in the 1960s, 1970s or 1980s were similar.

The risk reductions were also similar for epithelial and non-epithelial tumours. However, there was variation within the epithelial tumour types, as mucinous tumours were not affected much by oral contraceptive use. One of the main effects of oral contraceptives is to suppress ovarian activity, so protection against developing ovarian cancer is plausible. The exact mechanisms by which oral contraceptives cause the long-lasting protection shown by this study are, however, unknown.

These data indicate that oral contraceptives, first introduced in 1960 s, have already prevented some 200,000 ovarian cancers and 100,000 deaths from the disease. With increasing use of oral contraceptives in middle- and low-income countries, and the ageing population, the number of cancers prevented is likely to rise considerably in the next few decades.

In an accompanying Editorial, Richard Horton said that this study strengthened the case for oral contraceptives to be available over the counter. In an interview, Richard Peto, one of the lead authors of the group that published the study, said that the eventual reduction in ovarian cancer is bigger than any increase in other types of cancer, such as breast and cervical cancer, caused by the pill.

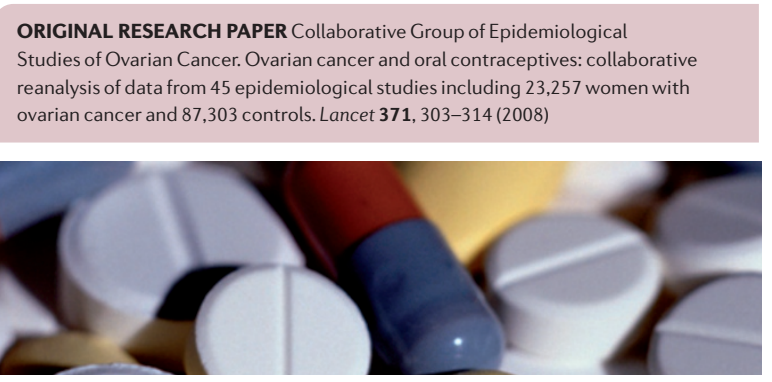

